\title{
Margin-based Active Online Learning Techniques for Cooperative Spectrum Sharing in CR Networks
}

\author{
Praveen Kumar K*, Eva Lagunas, Shree K Sharma, Satyanarayana Vuppala, \\ Symeon Chatzinotas, and Bjorn Ottersten \\ Interdisciplinary Centre for Security, Reliability and Trust \\ University of Luxembourg, Luxembourg \\ ${ }^{*}$ Corresponding author:praveen.korrai@uni.lu
}

\begin{abstract}
In this paper, we consider a problem of acquiring accurate spectrum availability information in the Cooperative Spectrum Sensing (CSS) based Cognitive Radio Networks (CRNs), where a fusion center collects the sensing information from all the sensing nodes within the network, analyzes the information and determines the spectrum availability. Although Machine Learning (ML) techniques have been recently applied to enhance the cooperative sensing performance in CRNs, they are mostly supervised learning based techniques and need a significant amount of labeled data, which is difficult to acquire in practice. Towards relaxing this requirement of large labeled data of supervised learning, we focus on Active Learning (AL), where the fusion center can query the label of the most uncertain cooperative sensing measurements. This is particularly relevant in CRN environments where primary user behavior changes in a quick manner. In this regard, we briefly review the existing AL techniques and adapt them to the considered CSS based CRNs. More importantly, we propose a novel margin based active on-line learning algorithm that selects the instance to be queried and updates the classifier by using the Stochastic Gradient Descent (SGD) technique. In this approach, whenever an unlabeled instance is presented, the proposed AL algorithm compares the margin of instance with a threshold to decide whether it should query a label or not. Supporting results based on numerical simulations show that the proposed method has significant advantages on classification and detection performances, and timecomplexity as compared to state-of-the-art techniques.

Key words- Active Learning, Cooperative Spectrum Sensing, Cognitive Radio Network.
\end{abstract}

\section{Introduction}

Due to the extensive proliferation of wireless applications and services, the wireless data traffic increases at an alarming rate and it is estimated to increase 10 folds by 2020 as compared to the current data traffic [1]. Though this increasing demand for wireless services has made the usable radio frequency spectrum a 
scarce and expensive resource, channel measurement field trails have illustrated that most of the time the radio spectrum is under-utilized by the licensed users, also known as primary users (PUs) [2]. In this context, Opportunistic Spectrum Access (OSA) is envisaged as a candidate technology to mitigate the spectrum scarcity by improving the utilization of spectrum [3]. In interweave CR networks (CRNs), the secondary users (SUs) can access the spectrum of the PUs opportunistically when the PU transmission is detected to be idle. Therefore, an efficient spectrum sensing mechanism is essential for the realization of efficient OSA.

In the above context, various spectrum sensing methods have been extensively discussed in the literature [4]. In this paper, we concentrate on energy detection (ED), which is one of the most widely accepted method as it is by far the cheapest and simplest option. However, due to the noise effect, the sensing efficiency of the ED method may be significantly degraded in the lower SNR regime [5]. Further, when the SUs are distributed in distinct locations, due to shadowing and severe multi-path fading, the hidden PU problem may occur. In this respect, the cooperative spectrum sensing (CSS) mechanism has been proposed to improve the sensing reliability by jointly processing the sensing information in a fusion center [6].

Machine learning techniques applied to CSS for CRNs have received significant research attention recently, e.g. [7-9]. In the context of interweave CR, the spectrum sensing problem reduces to a binary classification on the channel availability. Two major categories of learning methods to train the classifier are supervised and unsupervised learning methods. In supervised learning, first, the feature vectors (i.e data) should be labeled prior to training. Next, the feature vectors and its corresponding labels are fed into the classifier for training. Support vector machine (SVM) is one of the most popular supervised learning methods. SVM was exploited for spectrum sensing in $[7,8]$, showing its performance efficiency compared with the conventional ED based spectrum sensing. In contrast to the supervised learning algorithms, unsupervised learning algorithms do not require training (nor labeled data). Examples of unsupervised learning applied to CSS can be found in [8,9], where [9] has adopted a linear fusion rule for CSS and utilized the linear discriminant analysis to obtain linear coefficient weights, and [8] explored techniques such as Gaussian Mixture Models (GMM) and K-means clustering.

In the CSS problem, labeling data means knowing whether the PU is active or not at a particular time instant. In such a case, unlabeled data is abundant (i.e. spectrum sensing measurements) but labeling is challenging, as it requires feedback from the PUs to the SUs. To address this problem, Active Learning (AL) have been recently proposed, allowing the learning algorithms to dynamically query instances for labeling. AL has been applied in $[10,11]$ to learn the interference channel between the PU and SU in an underlay CRN. In addition, PUs behavior is not static, i.e. they may change from active to inactive at any time and vice-versa. In order to react fast to PU activity changes, in this paper, we investigate and analyze the applicability of online AL in ED-based CSS. 
First, we consider the perceptron based online AL technique [12] (also known as CBGZ algorithm), and we apply it to the proposed CSS problem. In this approach, when the classifier receives the energy measurements from the distributed SUs, it first predicts the availability of the channel from the energy instance, and then utilizes a random sampling approach to decide whether the label for such instance should be queried or not (i.e. if PU feedback is needed or not). If yes, the classifier acquires the true label from the system and follows the standard perceptron approach to update the classifier. Subsequently, the online passive aggressive AL (PAAL) algorithm [13] is considered for the proposed CSS problem. This algorithm follows the similar idea of CBGZ algorithm for querying, but utilizes an efficient PA learning strategy to update the classifier. However, the aforementioned AL algorithms depends on the uncertainty sampling strategy for querying, which is not accurate. To address this, we propose a margin based online AL algorithm with reduced threshold and Stochastic Gradient Descent (SGD) update. In this algorithm, margin based condition is utilized, which compares the margin of incoming instance with a threshold to decide whether it should query the class label or not. Finally, we compare and illustrate the performance of all these algorithms through numerical simulations, and also we compare them with the conventional supervised and unsupervised counterparts.

The rest of the paper is structured as follows. Section 2 presents the system model. We discuss the ML and AL based frameworks for the CSS in Section 3. The numerical evaluations of proposed frameworks are discussed in Section 4. Finally, conclusions are drawn in Section 5.

\section{System Model}

We consider an interweave $\mathrm{CRN}$, consisting of a PU, and multiple number of SUs where each SU is indexed by $m=1,2,3, \ldots, N$. The SUs collect energy measurements which are sent to a fusion center which jointly analyze them and determines the channel availability. The PU is placed at the coordinate $d^{P U}$ and the $m^{t h} \mathrm{SU}$ is placed at the coordinate $d_{m}^{S U}$ in the two-dimensional (2D) space. Further, all channels are considered to experience pathloss, and independent and identically distributed (IID) Nakagami-m fading. Therefore, the channel gain $h_{m}$ between the PU and the $m^{t h} \mathrm{SU}$ is expressed as

$$
h_{m}=P L\left(\left|d^{P U}-d_{m}^{S U}\right|\right) F_{m}
$$

where $P L(r)=r^{-\beta}$ represents the pathloss respective to distance between the $\mathrm{PU}$ and SU with the pathloss exponent $\beta$, and $F_{m}$ is the Nakagami-m fading component.

We assume the PU activity to vary over time in a random fashion, with 0.5 of probability of being in active state. Let $S$ denotes the state of PU. If the PU is in active state, then $S=1$, and otherwise $S=0$. Thus, the availability of channel is written as 


$$
Y=\left\{\begin{array}{l}
+1, \text { if } S=1 \\
-1, \text { if } S=0
\end{array}\right.
$$

where $Y=+1$ represents the unavailability of channel and $Y=-1$ represents the availability of channel.

We assume the cooperative sensing approach as shown in Fig. 1, in which each SU first determines the energy level of the signal transmitted by PU and reports it to a fusion center or classifier. Further, based on the sensed energy levels reported by all SUs, the fusion center or classifier estimates the availability of spectrum.

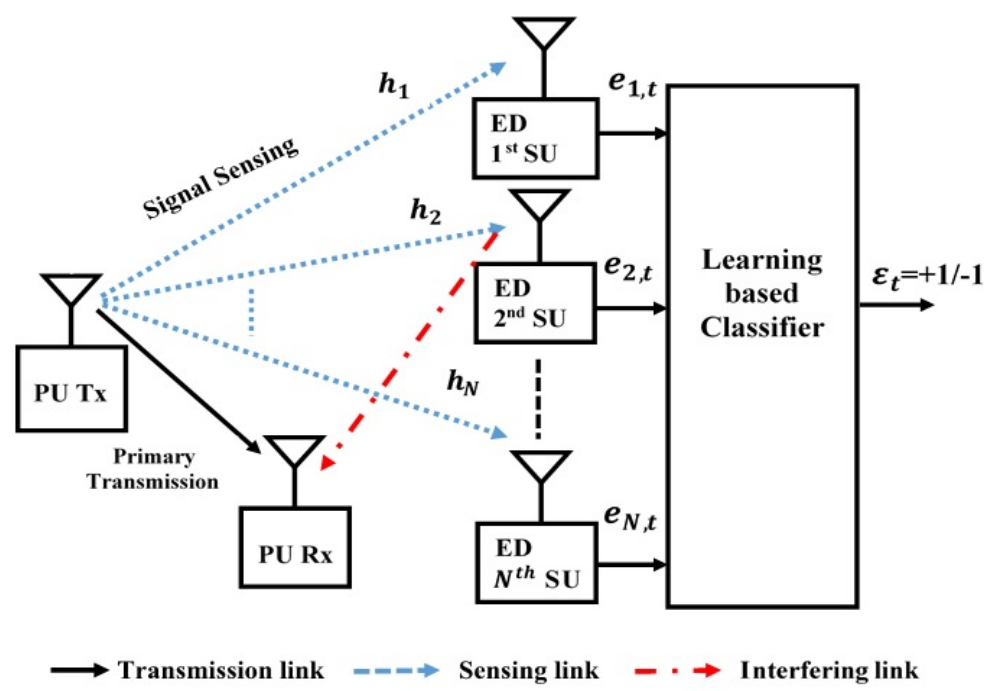

Fig. 1. Learning Based Cooperative Spectrum Sensing Model

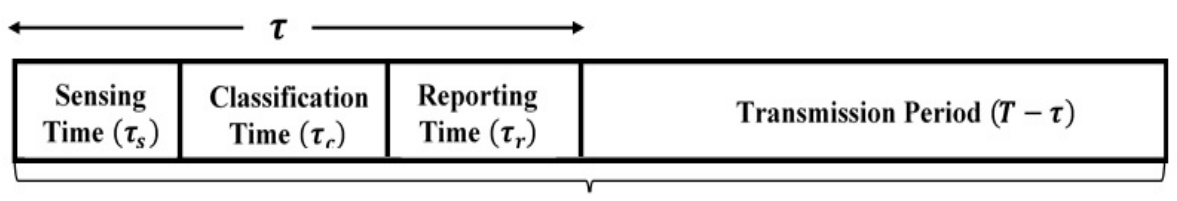

Frame Duration $(T)$

Fig. 2. Frame structure of SU transmission

In order to perform the energy detection, SUs require to compute the energy level of primary transmitted signal for a certain period of time. The frame structure of the SU is depicted in Fig. 2. Each frame consists the deciding time $\tau$ (i.e., 
a combination of sensing time $\left(\tau_{s}\right)$, classification time $\left(\tau_{c}\right)$, and reporting time $\left.\left(\tau_{r}\right)\right)$ and a transmission duration $(T-\tau)$. Let $W$ be the assigned bandwidth for the signal transmission and $\tau_{s}$ be the spectrum sensing duration; then, the enegry detector senses $W \tau_{s}$ complex samples during the sensing time. Therefore, the $i^{\text {th }}$ signal sample sensed by the $m^{\text {th }} \mathrm{SU}$ can be expressed as

$$
X_{m}(i)=S h_{m} y(i)+N_{m}(i)
$$

where $y(i)$ denotes the transmitted signal by the PU, and $N_{m}(i)$ is the thermal noise at the $m^{t h} \mathrm{SU}$. Thus, at the output of energy detector of $m^{\text {th }} \mathrm{SU}$, the total estimated energy of received signal samples normalized by noise spectral density can be expressed as [8]

$$
e_{m, l}=\frac{2}{\eta} \sum_{i=1}^{W \tau_{s}}\left|X_{m}(i)\right|^{2}, \quad m=1,2, . ., N, \text { and } l=1,2, \ldots, L
$$

where $\eta=\mathbb{E}\left\{\left|N_{m}(i)\right|^{2}\right\}$ is the power spectral density of noise. The complete set of sensed energies from $N$ SUs at a given time $t$ can be written in vector form as follows,

$$
\mathcal{E}_{t}=\left[e_{1, t} e_{2, t} \ldots e_{N, t}\right]^{T}
$$

where $e_{m, t}$ is equivalent to (4) assuming that we collect energy measurements at each $t^{t h}$ frame. The fusion center receives $\mathcal{E}_{t}$ sequentially and has to make a decision on the channel availability after each reception of energy measurements. If

the channel is identified as idle, the information is transmitted for the remaining time of the frame.

\section{Learning Based Frameworks for Spectrum Sensing}

ML based frameworks have already been adapted for CSS applications in the literature [8] and shown that supervised learning algorithms are efficient for these applications. However, in supervised learning tasks, a huge data set with labels are required to train the model. The gathering of data and labeling for it is very time-consuming and costly in these specific applications. To address this problem, in this section, we develop online AL algorithms based CSS schemes to predict the channel availability. First, we exploit the margin based online AL algorithms such as CBGZ [12] and PAAL [13] and we adapt them to fit in the proposed CSS problem. Next, we propose a reduced threshold and SGD update based online AL algorithm for CSS.

\subsection{The CBGZ Algorithm}

In an attempt to minimize the number of requested labeled queries and so the feedback from the PU, the CBGZ algorithm makes use of an improved Perceptron-type of algorithm, which considers a margin-based filtering rule. The application of CBGZ in the problem at hand is as follows. After receiving the 
energy measurement vector $\mathcal{E}_{t}$, the algorithm queries the PU for the label with a probability of $d /(d+|\hat{b}|)$, where $d$ is a constant and $\hat{b}$ represents the uncertainty of the received instance, and it is computed as a margin of the received measurement instance with respect to the present hypothesis. Next, the algorithm's prediction is compared with the feedback received by the PU and if does not match, the standard Perceptron update process is invoked. The summary of the CBGZ algorithm for CSS is presented in Algorithm 1.

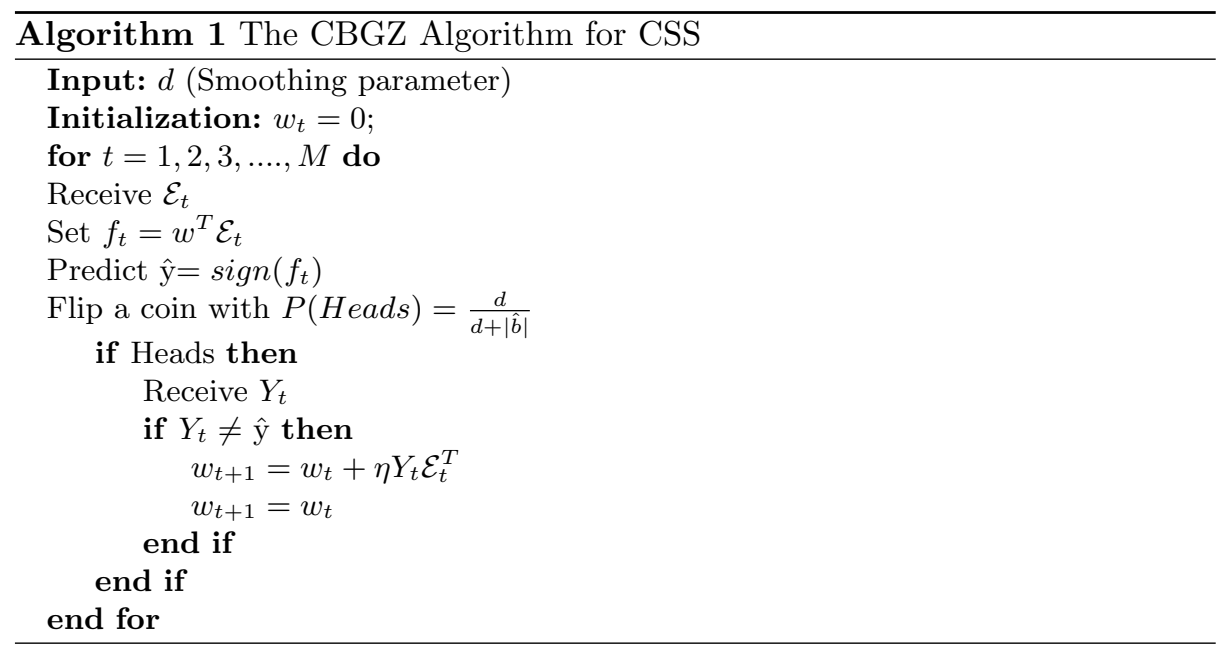

\subsection{Passive Aggressive AL for CSS}

In this Section, we consider the PAAL algorithm for the proposed CSS problem. As in CBGZ, this algorithm consists in two stages: (i) querying process and (ii) classifier updating process. In the first stage, the algorithm follows the same approach of margin-based AL.

First, the algorithm estimates the uncertainty or margin of the incoming energy instance using the current classifier i.e $p_{t}=w_{t} \cdot \mathcal{E}_{t}$. Next, it draws a Bernoulli random variable $Z_{t} \in\{0,1\}$ with probability $C /\left(C+\left|p_{t}\right|\right)$ to decide whether the label should be requested or not. If $Z_{t}=0$, the class label is not queried and the classifier is not updated. Otherwise, the class label of the incoming energy instance is queried at the PU, and unlike the previous margin-based AL algorithm that updates the classifier only whenever a misclassification occurs, the PAAL algorithm updates the classifier whenever the value of loss function is not zero. Then, the classifier updates as $w_{t+1}=w_{t}+\xi_{t} y_{t} \mathcal{E}_{t}$, where $\xi_{t}$ is the step-size estimated as

$$
\xi_{t}=\left\{\begin{array}{cc}
l_{t}\left(w_{t}\right) /\left(\left\|\mathcal{E}_{t}\right\|^{2}+1 /(2 A)\right) & ; \text { PAA-3 } \\
\min \left(C, l_{t}\left(w_{t}\right) /\left(\left\|\mathcal{E}_{t}\right\|^{2}\right.\right. & ; \text { PAA-2 } \\
l_{t}\left(w_{t}\right) /\left(\left\|\mathcal{E}_{t}\right\|^{2}\right) & ; \text { PAA-1 }
\end{array}\right.
$$


The complete procedure of the PAAL algorithm is summarized in Algorithm 2 .

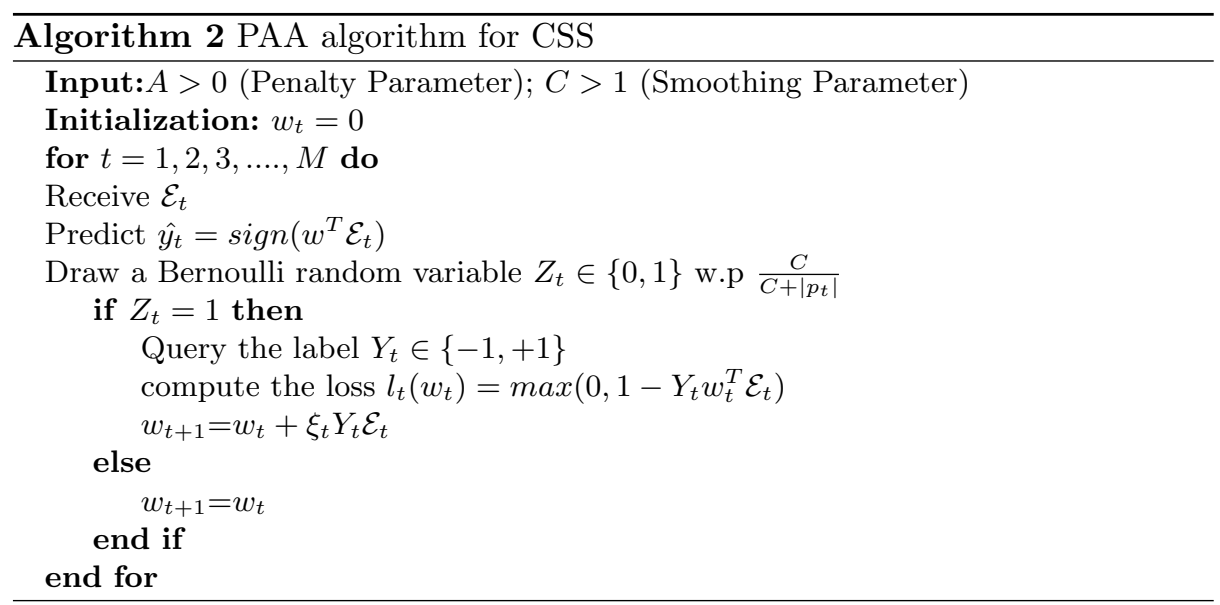

\subsection{Proposed Margin-Based Method with Reduced Threshold and SGD Update}

Both PAAL and CBGZ algorithms utilize a computationally complex uncertain sampling strategy for querying. Hence, in order to avoid the uncertainty sampling step, in this section, we propose a threshold based querying strategy algorithm for CSS. The proposed algorithm comprises two steps: (i) selection of instance, and (ii) Update process of the classifier. The margin of $\mathcal{E}_{t}$ is computed as $W_{t}^{T} \mathcal{E}_{t}$, and that is compared with the existing threshold $C_{t}$. If the margin is lower than the existing threshold, then the classifier $w_{t}$ is updated using the SGD step. Finally, we determine the upper bound of estimation error, which is used in the update of threshold $C_{t}$. The complete procedure is summarized as Algorithm 3 .

Computation of Threshold $\left(C_{t}\right)$ : Assume $w_{*}$ is an optimal classifier with $v$ error rate. Here, our objective is to find the condition that for a given energy instance, the classifier $w_{t}$ should provide the same prediction as the optimal one $w_{*}$. This condition reveals if a certain energy instance is not useful to our classifier, and thus if it can excluded by the threshold.

For each iteration $t$, consider $\phi_{t}$ is the angle of $w_{t}$ and $w_{*}$. For every energy instance $\mathcal{E}_{t}, w_{*}\left(\mathcal{E}_{t}\right)$ and $w_{t}\left(\mathcal{E}_{t}\right)$ predict the same sign if the margin of the instance $\left|w_{t}^{T} \mathcal{E}_{t}\right|$ is greater than the angle of classifier $\phi_{t}$. If $\left|w_{t}^{T} \mathcal{E}_{t}\right| \leq \phi_{t}$, for instance $\left\{\mathcal{E}_{t}: \operatorname{sign}\left(h_{t}\left(\mathcal{E}_{t}\right)\right) \neq \operatorname{sign}\left(h_{*}\left(\mathcal{E}_{t}\right)\right)\right\}$. Therefore, we have to make $C_{t} \geq \phi_{t}$.

The upper bound for $\phi_{t}$ can be expressed as

$$
P\left\{\left|w_{t}^{T} \mathcal{E}_{t}\right| \leq \phi_{t}\right\} \leq b_{a} P\left\{\mathcal{E}_{t}: \operatorname{sign}\left(h_{t}\left(\mathcal{E}_{t}\right)\right) \neq \operatorname{sign}\left(h_{*}\left(\mathcal{E}_{t}\right)\right)\right\}
$$

where $b_{a}$ is a constant and $P$ is the instances distribution. We have $P\left\{E_{t}\right.$ : $\left.\operatorname{sign}\left(h_{t}\left(\mathcal{E}_{t}\right)\right) \neq \operatorname{sign}\left(h_{*}\left(\mathcal{E}_{t}\right)\right)\right\} \leq 2 v+\operatorname{Er}_{t}$ and $\phi_{t} \leq b_{c} P\left\{\left|w_{t}^{T} \mathcal{E}_{t}\right| \leq \phi_{t}\right\}$. Finally, 
by using equations, we get $\phi_{t} \leq B\left(2 v+E r_{t}\right)$. where $B=b_{a} b_{c}$. Therefore, we set the threshold to be $C_{t}=B\left(2 v+E r_{t}\right)$.

Classifer update using SGD: SGD updates the classifier as

$$
w_{t+1}=w_{t}-\epsilon_{t} \frac{\partial}{\partial w} l\left(w^{T} \mathcal{E}_{t}, y_{t}\right)
$$

where $l\left(w^{T} E_{t}\right)$ is the hinge loss. After some mathematical manipulations, we obtain the following SGD update policy,

$$
w_{t+1}= \begin{cases}w_{t}+\frac{1}{\sqrt{t}} Y_{t} \mathcal{E}_{t}, & \text { if } Y_{t} w^{T} \mathcal{E}_{t}<1 / \sqrt{t}, \\ w_{t} & \text { otherwise }\end{cases}
$$

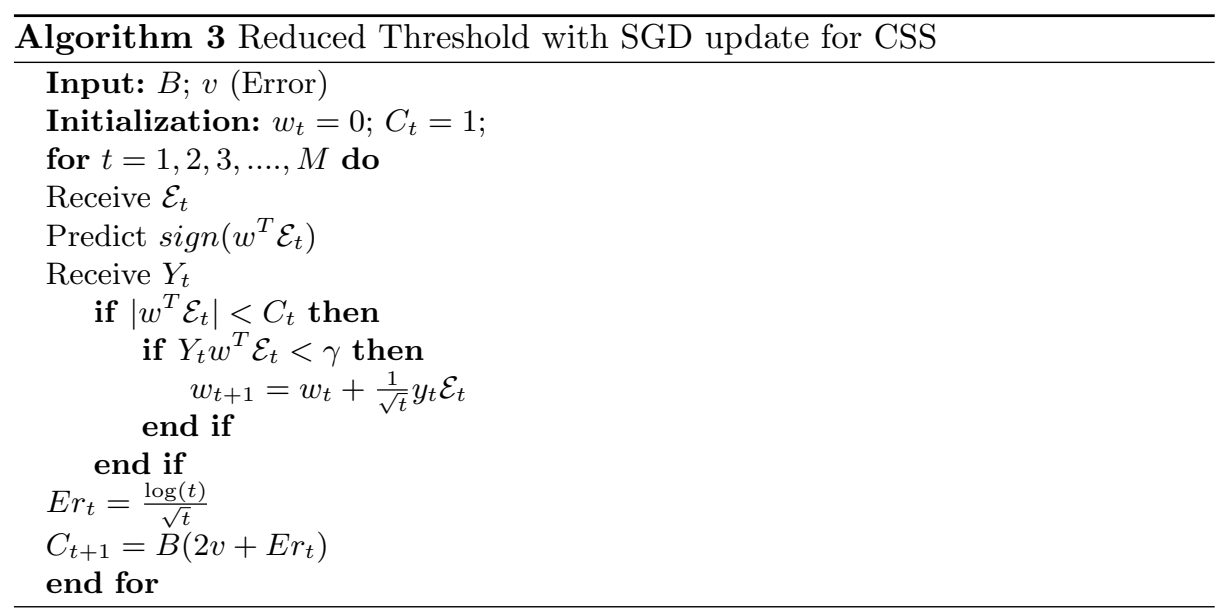

\section{Numerical Evaluations}

To show the benefits of the proposed scheme, in this section, we show the empirical performance of the considered online AL algorithms for CSS tasks in CRNs and we compare them with state-of-the art ML algorithms such as SVM and $\mathrm{K}$-means in terms of miss-classification rate and time-complexity.

\subsection{Simulation Parameters}

We consider a wireless network with a PU that is located at coordinates $(500 \mathrm{~m}$, 0 ) and nine SUs involving in CSS that are located in a 3-by-3 grid topology in the area of $2000 \mathrm{~m} \times 2000 \mathrm{~m}$ as depicted in Fig. 3. Further, we assign $5 \mathrm{MHz}$ bandwidth $W$ for each transmission, each frame duration is $100 \mathrm{~ms}$, sensing duration $\tau_{s}$ is assumed as $10 \mathrm{~ms}$, the PL exponent is 3 , and the additive white Gaussian noise (AWGN) is considered.

In the on-line AL algorithms, there are some other parameters such as optimal error $v$ and constant $B$ in Algorithm 3, smoothing parameter $C$ and penalty 


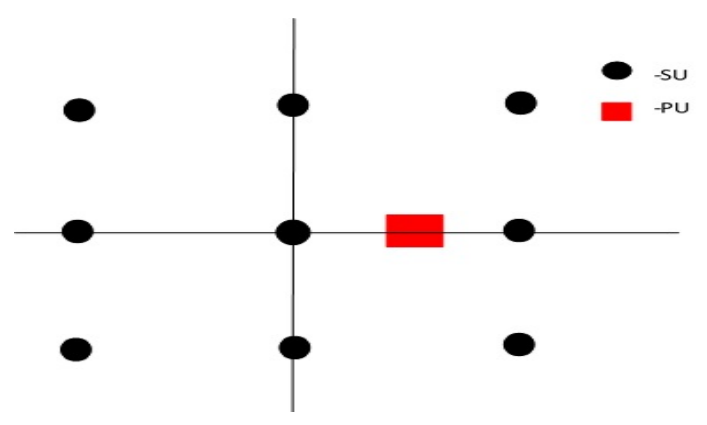

Fig. 3. Example of network model with $N=9$ SUs

parameter $A$ in Algorithm 2, learning parameter $\eta$ and constant $d$ to compute the probability of sampling in Algorithm 1, which are all estimated through the cross validation procedure. We run the experiment over a total of 1024 frames. All the experiments are performed and averaged over 5 independent Monte-Carlo runs, each with a random PUs behavior resulting in a different permutation of the energy dataset within the 1024 instances.

For the experiments related to the passive learning SVM algorithm, we use 1024 energy instances for the training and 1024 energy instances for the testing. For the experiments related to the passive learning K-means algorithm, we compute the classifier assuming 1024 non-labeled energy instances and next we proceed to the testing phase with 1024 different energy instances.

In the testing of online AL algorithms, all energy instances come one by one in a sequential manner. If the incoming energy instance is identified as a most informative, then the querying process is executed to obtain the true class label. The latter is assumed to be provided by the PUs in a form of 1-bit feedback. After receiving the true channel availability, that energy instance is added to training set. The same process is continued till to end of the experiment.

\subsection{Results and Discussions}

Fig. 4 illustrates the performance of SVM, K-means and on-line AL based CSS schemes in terms of mis-classification rate. From the results, it is observed that the CSS schemes based on-line AL algorithms outperform the conventional SVM and K-means based CSS schemes. This is due to fact that whenever the misclassification occurs, the considered online AL algorithms update the classifier according to the margin of the incoming energy instance. This classifier updating process helps to reduce the further classification errors. Also, we notice from the results in Fig. 4 that the CSS scheme based on the proposed online AL algorithm with reduced threshold and SGD update outperforms the online PAAL algorithms and the state-of-the art CBGZ AL algorithm based CSS schemes. The proposed algorithm constantly shows almost 10 percentage of lower mis- 


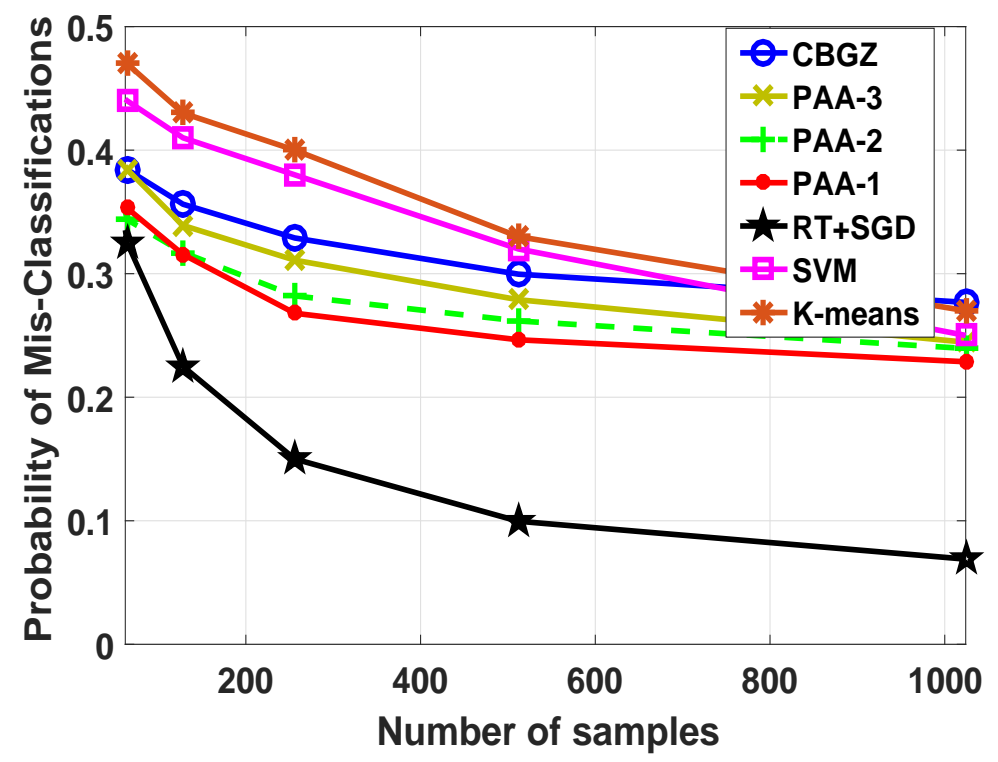

Fig. 4. Evaluation of CSS schemes based on on-line AL methods and conventional ML methods.

classification rate compared to PAAL algorithms and more than 15 percentage of lower misclassification rate compared to CBGZ algorithm.

We also provide the detailed comparison of the supervised, unsupervised and online AL algorithms in Table 1 and Table 2. As can be seen form Table 1, both the supervised and unsupervised algorithms SVM and K-means are interestingly illustrating the almost same performance using the size of 1024 data samples for training and for the classifier computation, respectively. However, the time-complexity of K-means algorithm is very high as compared to the SVM algorithm. Also, from Table 2, it is observed that the proposed RT+SGD AL algorithm shows better performance compared to other AL algorithms in terms of both misclassification and run time.

Table 1. Percentage of Misclassifications and Time complexity

\begin{tabular}{|l|l|l|}
\hline AL algorithms & $\begin{array}{l}\text { Percentage of } \\
\text { Misclassifications }\end{array}$ & Run Time (s) \\
\hline SVM & 25.4 & 0.0453 \\
\hline K-means & 28.2 & 0.0953 \\
\hline
\end{tabular}




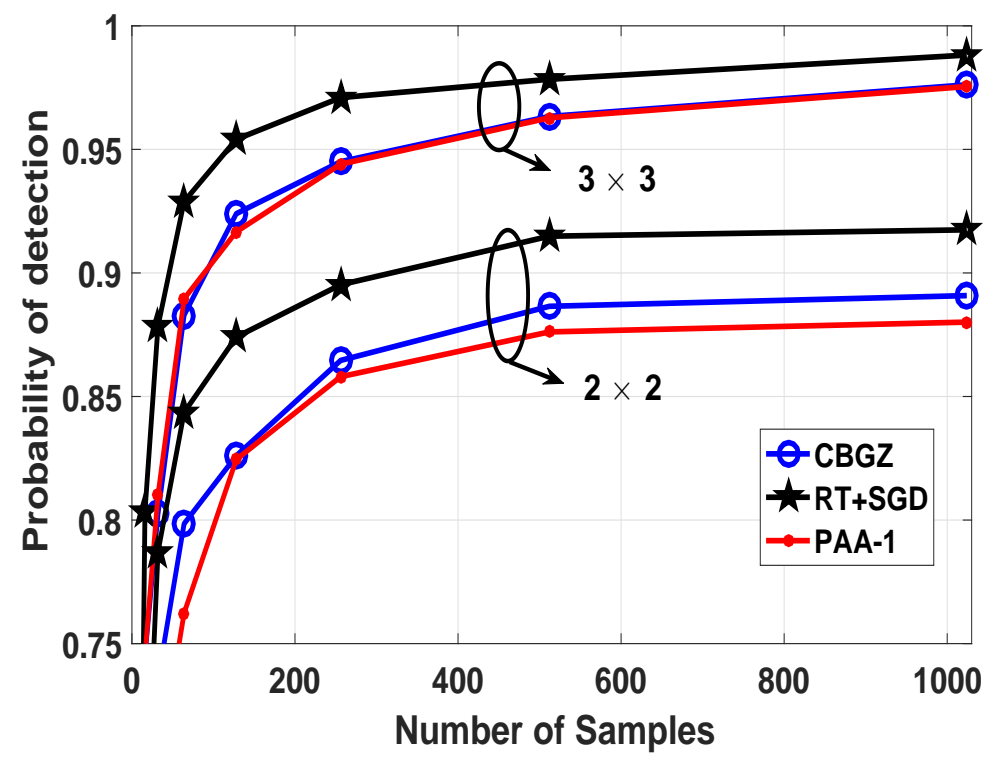

Fig. 5. Probability of detection of CSS schemes based on on-line AL methods

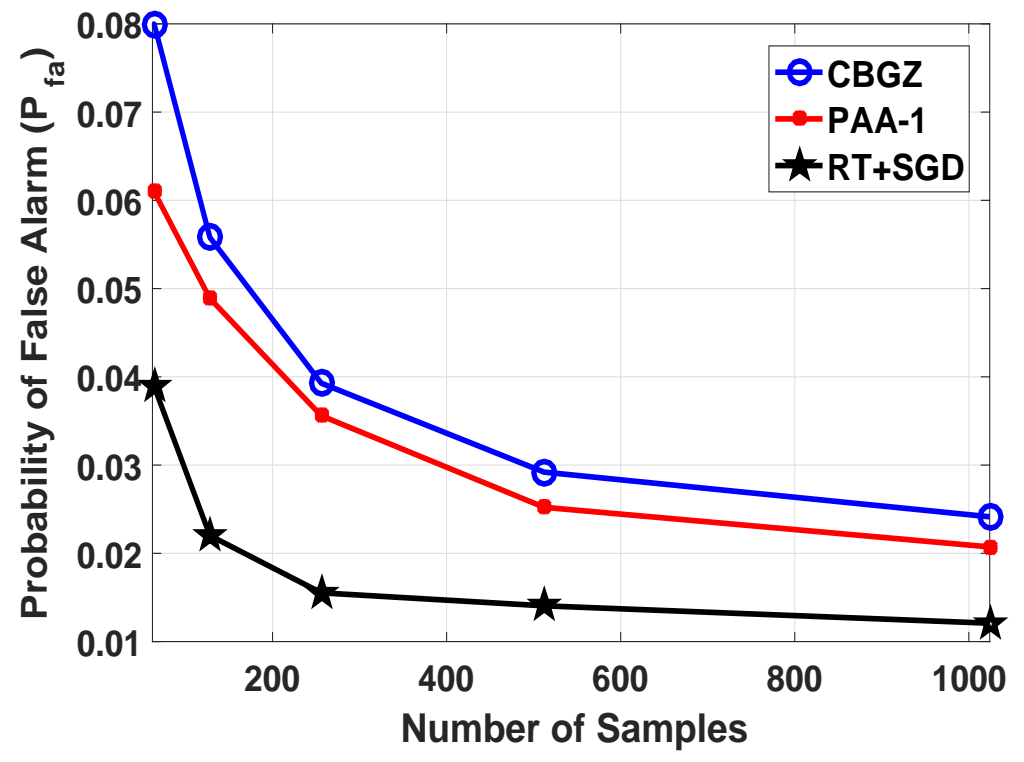

Fig. 6. Probability of false alarm of CSS schemes based on on-line AL methods 
In Fig. 5, we show the performance of the proposed online AL based CSS schemes in terms of probability of detection when $2 \times 2$ SUs (i.e 4 SUs) and $3 \times 3$ SUs (i.e 9 SUs) cooperate in CSS. From the obtained results, we observe that the proposed online AL algorithm with reduced threshold and SGD update (SGD+RT) algorithm outperforms the other AL algorithms with almost 3 percentage of detection rate. Also, in Table 3, we provide the percentage of misclassification and the time duration to identify the busy or idle PU channel state using the AL classifiers with 4 and 9 SUs in cooperation for CSS. We also observe from the results that with 9 cooperating SUs, the detection rate of the AL based algorithms improves by increasing the number of instances, as expected. For example, after 200 energy instances the proposed CSS scheme based on the SGD $+\mathrm{RT}$ algorithm achieves almost +7 percentage of detection rate with 9 number of SUs cooperation as compared to 4 number of SUs cooperation.

Table 2. Percentage of Misclassifications and Time complexity of online AL algorithms

\begin{tabular}{|l|l|l|}
\hline AL algorithms & $\begin{array}{l}\text { Percentage of } \\
\text { Misclassifications }\end{array}$ & Run Time (s) \\
\hline CBGZ & 28.6 & 0.0423 \\
\hline PAA-3 & 24.6 & 0.0311 \\
\hline PAA-2 & 23.8 & 0.0301 \\
\hline PAA-1 & 23.1 & 0.0295 \\
\hline SGD+RT & 7.5 & 0.0046 \\
\hline
\end{tabular}

We show the performance comparison of AL algorithms based CSS schemes in terms of probability of false alarm $\left(P_{f a}\right)$ in Fig. 6. From the results, it is observed that the proposed RT+SGD algorithm shows the less false alarm rate as compared to the other two algorithms. In Fig. 6, we provide the performance comparison of the proposed AL algorithms in terms of the normalized throughpu. Using the probability of false alarm, the normalized throughput of the secondary network is expressed as [14]

$$
T P_{\text {norm }}=\frac{T-\tau}{T}\left(1-P_{f a}\right),
$$

As can be seen from the results that the proposed RT+SGD algorithm obtains the highest normalized throughput.

Table 3. Percentage of Misclassifications and Time complexity of 2 and3 SUs cooperation

\begin{tabular}{|l|l|l|l|l|}
\hline AL algorithms & Percentage of Misclassifications & Run Time (s) \\
\hline & $2 \times 2$ & $3 \times 3$ & $2 \times 2$ & $3 \times 3$ \\
\hline CBGZ & 32.7 & 28.6 & 0.0411 & 0.0423 \\
\hline PAA-1 & 25.4 & 23.1 & 0.0290 & 0.0295 \\
\hline SGD+RT & 13.3 & 7.5 & 0.0038 & 0.0046 \\
\hline
\end{tabular}




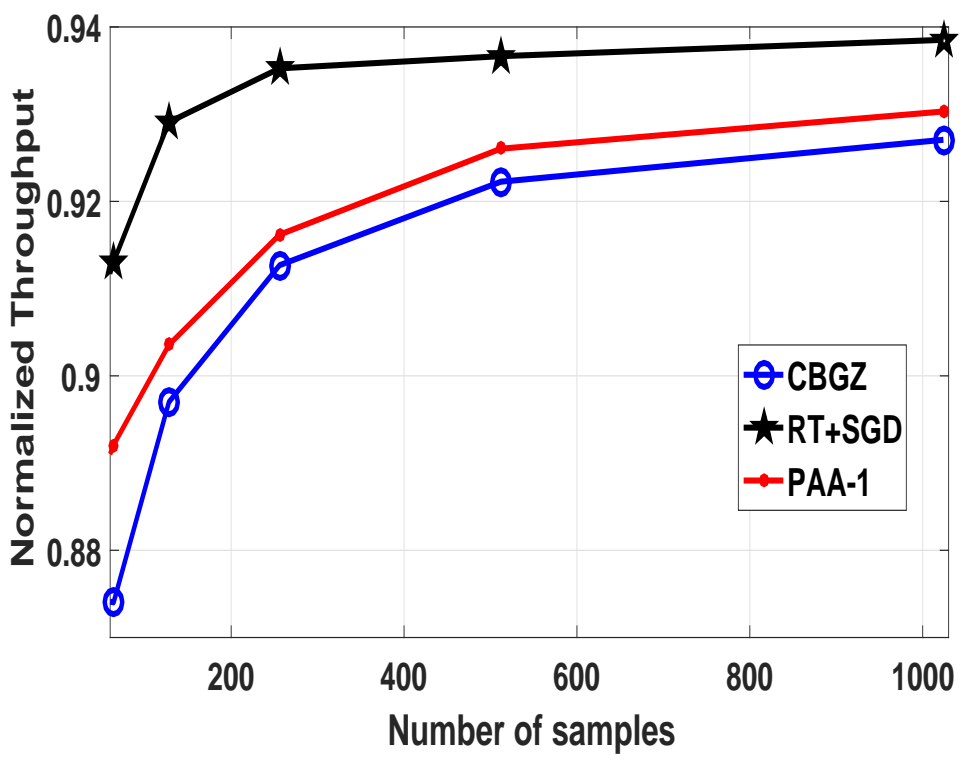

Fig. 7. Throughput analysis of on-line AL based CSS methods

\section{Conclusions}

In this paper, we have formulated online AL algorithms for CSS. In particular, first, we have utilized the state-of-the-art CBGZ algorithm and the PAAL algorithm, wherein uncertainty sampling with some probability was utilized as a query strategy. To overcome the complexity of the aforementioned sampling strategy, next, we proposed a margin based online AL algorithm with reduced threshold and SGD update for CSS, wherein the margin of incoming energy instance was compared with a threshold to decide whether the class label needs to be queried or not. After querying the sample, SGD was used to update the classifier. Finally, we have provided a comparison and analysis of the performance of the different on-line AL algorithms in terms of probability of detection, misclassifications, time complexity and normalized throughput. The proposed online AL algorithm with reduced threshold and SGD update has achieved the highest detection rate, normalized throughput and the lowest misclassification and run time as compared to the state-of-the art CBGZ AL algorithm and PAAL algorithms.

\section{Acknowledgment}

This work has received partial funding from the European Research Council (ERC) under the European Unions Horizon H2020 research and innovation 
programme (grant agreement No 742648), and from the Luxembourg National Research Fund (FNR) in the framework of the AFR research grant entitled "Learning-Assisted Cross-Layer Optimization of Cognitive Communication Networks".

\section{References}

1. CISCO, "Cisco visual networking index: Global mobile data traffic forecast update, 2015-2020," Feb. 2016.

2. M. Lopez-Benitez and F. Casadevall, "Spectrum Occupancy in Realistic Scenarios and Duty Cycle Model for Cognitive Radio," Adv. Electron.Telecommun., vol. 1, no. 1, pp. 26-34, Apr. 2010.

3. Y. Lee, "Opportunistic Spectrum Access in Congnitive Networks," Electronics Letters, vol. 44, no. 17, pp. 1022-1024, Aug. 2008.

4. T. Yucek and H. Arslan, "A survey of spectrum sensing algorithms for cognitive radio applications," IEEE Communications Surveys Tutorials, vol. 11, no. 1, pp. 116-130, 2009.

5. E. Axell, G. Leus, E.G. Larsson, and H.V. Poor, "Spectrum sensing for cognitive radio: State-of-the-art and recent advances," IEEE Signal Process. Mag., vol. 29, no. 3, pp. 101-116, May 2012.

6. R. Saifan, I. Jafar, and G. Al-Sukkar, "Optimized Cooperative Spectrum Sensing Algorithms in Cognitive Radio Networks," The Computer Journal, vol. 60, no. 6, pp. 835-849, Jun. 2017.

7. D. Zhang and X. Zhai, "SVM-Based Spectrum Sensing in Cognitive Radio," Int. Conf. Wireless Commun., Net. and Mobile Computing (WICOM), Wuhan, China, 2011.

8. K.M. Thilina, K.W. Choi, N. Saquib, and E. Hossain, "Machine Learning Techniques for Cooperative Spectrum Sensing in Cognitive Radio Networks," IEEE J. Sel. Areas Commun., vol. 31, no. 11, pp. 2209-2221, Nov. 2013.

9. K.W. Choi, E. Hossain, and D.I. Kim, "Cooperative Spectrum Sensing Under a Random Geometric Primary User Network Model," IEEE Trans. Wireless Commun., vol. 10, no. 6, pp. 1932-1944, Jun. 2011.

10. A. Tsakmalis, S. Chatzinotas, and B. Ottersten, "Interference Constraint Active Learning with Uncertain Feedback for Cognitive Radio Networks," IEEE Trans. Wireless Commun., vol. 16, no. 7, pp. 4654-4668, Jul. 2017.

11. A. Tsakmalis, S. Chatzinotas, and B. Ottersten, "Constrained Bayesian Active Learning of Interference Channels in Cognitive Radio Networks," IEEE J. Sel. Topics Signal Process., vol. 12, no. 1, pp. 6-19, Feb. 2018.

12. N. Cesa-Bianchi, C. Gentile, and L. Zaniboni, "Worst-Case Analysis of Selective Sampling for Linear-Threshold Algorithms," Advances in Neural Information Processing Systems Conference, 2004.

13. J. Lu, P. Zhao, and S.C.H. Hoi, "Online Passive-Aggressive Active learning," Machine Learning, vol. 103, no. 2, pp. 141-183, May 2016.

14. Y Liang, Y. Zeng, Peh E. C. Y., and Hoang A.T., "Sensing-throughput tradeoff for cognitive radio networks," IEEE Transactions on Wireless Communications, vol. 7 , no. 4, pp. 1326-1337, 2008. 